\title{
Induction in vitro de l'enracinement de microboutures d'Acacia tortilis subsp. raddiana par traitement transitoire à l'auxine
}

\author{
Djibril Sanéa, $^{a,}$, Alain Borgel ${ }^{b}$, Marie-Hélène Chevallier ${ }^{c}$ et Yaye Kène Gassama-Dia ${ }^{a}$ \\ a Département de Biologie Végétale, Université Cheikh Anta Diop, BP 5005, Dakar-Fann, Sénégal \\ b IRD, Laboratoire GeneTrop, BP 5045, 34032 Montpellier Cedex 1, France \\ ${ }^{\mathrm{c}}$ Cirad Forêt, TA 10/C, 34398 Montpellier Cedex 5, France
}

(Reçu le 29 décembre 1999; accepté le 4 décembre 2000)

\begin{abstract}
Résumé - Chez Acacia tortilis raddiana, l'application transitoire d'auxine (acide indol butyrique (AIB) ou acide naphtalène acétique (ANA) à 10 ou $20 \mathrm{mg} \mathrm{L}^{-1}$ ) combinée ou non avec une cytokinine (kinétine à $0,01 \mathrm{mg} \mathrm{L}^{-1}$ ) pendant 10 ou 20 jours a permis d'améliorer l'enracinement (nombre et longueur moyenne des racines) après le transfert des microboutures sur un milieu Lloyd et McCown (WPM) dépourvu d'hormone. Le pourcentage des microboutures enracinées sur milieu d'expression est de $56 \%$ en moyenne et atteint $80 \%$ avec un traitement inductif à $10 \mathrm{mg} \mathrm{L}^{-1} \mathrm{~d}$ 'AIB. Les mesures effectuées après 28 jours de culture ont montré que la nature de l'auxine utilisée pour induire la rhizogenèse a une influence significative aussi bien sur la fréquence de l'enracinement que sur la morphologie du système racinaire qui se met en place. Avec l'ANA les racines sont peu nombreuses ( 1 à 5 racines par bouture) et robustes et orthotropes ( 4,5 à $5 \mathrm{~cm}$ en moyenne) dans $80 \%$ des cas et avec l'AIB, elles sont très nombreuses (10 à 15 racines par bouture) et fines et plagiotropes (3 à $3,7 \mathrm{~cm}$ ) dans $68 \%$ des cas.
\end{abstract}

Acacia tortilis raddiana / microbouture / auxine / induction / rhizogenèse

\begin{abstract}
Transient auxin treatment for in vitro rooting of microcuttings of Acacia tortilis subsp. raddiana. An auxin in vitro pretreatment (IBA or NAA at 10 to $\left.20 \mathrm{mg} \mathrm{L}^{-1}\right)$ combined with a low concentration of Kinetin $\left(0.01 \mathrm{mg} \mathrm{L}^{-1}\right)$ during 10 or 20 days allowed to improve the rooting (number and root length) of Acacia tortilis raddiana microcuttings after transfer on Lloyd and Mc Cown medium (WPM) without hormone. After microcuttings transfer in expression medium, 56\% are rooted and the rooting frequency can reach $80 \%$ after pretreatment with IBA at $10 \mathrm{mg} \mathrm{L}^{-1}$. Measurements made after 28 days show that the auxin type used for inducing rhizogenesis has a significant effect, both on rooting percentage and rooting system morphology. For $80 \%$ of plantlets, the number of roots is low with NAA (1 to 5) but roots are strong and orthotropic ( 4.5 to $5 \mathrm{~cm}$ on average) when with IBA roots are numerous (10 to 15$)$, but thin and plagiotropic ( 3 to $3.7 \mathrm{~cm}$ ) for $68 \%$ of rooted plantlets.
\end{abstract}

Acacia tortilis raddiana / microcutting / auxin / induction / rhizogenesis

* Correspondance et tirés-à-part

Tél. (221) 8250443 ; e-mail : djisane@ refer.sn 


\section{INTRODUCTION}

Acacia tortilis subsp. raddiana (A. raddiana) est une espèce ligneuse arborée de la famille des légumineuses. Très résistantes à la sécheresse, les populations d'A. raddiana forment généralement la limite des zones arborées du désert [4]. Au Sahel, cette espèce à usages multiples est souvent utilisée comme essence de reboisement en raison de son potentiel fertilisant lié à la symbiose fixatrice d'azote atmosphérique [6].

Espèce allogame et hétérozygote, l'évaluation de la valeur génétique d'A. raddiana passe par la création de clones, matériel homogène nécessaire aussi bien pour étudier la valeur génétique des individus et les interactions génotypes/milieu que pour la production en masse de génotypes bien adaptés aux conditions édapho-climatiques de la zone sahélienne [9]. La multiplication végétative n'étant pas naturelle chez cette espèce, les meilleures conditions de clonage in vitro par microbouturage ont été recherchées [3].

L'enracinement correspond à une étape essentielle mais difficile de la multiplication végétative in vitro des ligneux. Si l'aptitude à l'enracinement in vitro apparaît généralement élevée sur le matériel végétal juvénile, celle-ci décroît très rapidement au fil des subcultures de microboutures [13]. À partir de nœuds de jeunes plants d'A. raddiana $90 \%$ de microboutures enracinées ont été obtenues à la première culture et seulement $2 \%$ après une deuxième subculture de deux mois sur le même milieu en présence d'acide indole acétique (AIA) ou d'acide indole butyrique (AIB) [3]. Par ailleurs sur des microboutures issues directement de nœuds cotylédonaires d'A. raddiana une application permanente d'AIB ou d'acide naphtalène acétique (ANA) a permis d'observer des fréquences de $20 \%$ à $65 \%$ d'enracinement [11]. L'application d'hormones est indispensable et des auxines comme l'AIB ou l'ANA appliquées de manière transitoire permettraient d'améliorer l'enracinement d'espèces forestières cultivées in vitro $[2,16]$.

Cet article présente les résultats obtenus concernant l'action de deux auxines (AIB et ANA) couplées ou non avec une cytokinine (Kinétine) appliquées de manière transitoire (10 jours ou 20 jours) sur l'enracinement et la croissance de microboutures d'A. raddiana.

\section{MATÉRIEL ET MÉTHODES}

Les semences d'A. raddiana ont été collectées au Sénégal dans la région de Saint-Louis, au village de Mbari- go. Les graines ont été scarifiées à $\mathrm{l}^{\prime} \mathrm{H}_{2} \mathrm{SO}_{4}$ à $95 \%$ pendant 2 heures. Après rinçage à l'eau distillée stérile, elles ont été mises à germer en conditions aseptiques sur un milieu simple de germination composé des macro-éléments et micro-éléments de Murashige et Skoog [10] et solidifié par l'agar à $0,7 \%$.

Après un mois de culture, les plantules ont été entièrement découpées en segments uninodaux de $10 \mathrm{~mm}$ de longueur en excluant les segments contenant le nœud cotylédonaire. Les microboutures ont été cultivées pendant deux mois sur un milieu de multiplication composé des macro-éléments du Woody Plant Medium (WPM) [8], des micro-éléments de MS, des vitamines de Nitsch et Nitsch (N \& N) [12], de saccharose à $20 \mathrm{~g} \mathrm{~L}^{-1}$, d'agar à $0,6 \%$, de zéatine à $0,1 \mathrm{mg} \mathrm{L}^{-1}$ et d'AIB à $1,2 \mathrm{mg} \mathrm{L}^{-1}$. Les pousses ainsi obtenues ont été découpées en microboutures uninodales de $10 \mathrm{~mm}$ transférées sur les milieux d'induction à l'enracinement composés des macro-éléments du WPM, des micro-éléments de MS, des vitamines de $\mathrm{N} \& \mathrm{~N}$, de saccharose à $10 \mathrm{~g} \mathrm{~L}^{-1}$, d'agar à $0,7 \%$, avec six combinaisons de régulateurs de croissance (ANA $10 \mathrm{mg} \mathrm{L}^{-1}$; ANA $10 \mathrm{mg} \mathrm{L}^{-1}+$ kinétine $0,01 \mathrm{mg} \mathrm{L}^{-1}$; ANA $20 \mathrm{mg} \mathrm{L}^{-1}$; AIB $10 \mathrm{mg} \mathrm{L}^{-1}$; AIB $10 \mathrm{mg} \mathrm{L}^{-1}+$ kinétine $0,01 \mathrm{mg} \mathrm{L}^{-1}$ et AIB $20 \mathrm{mg} \mathrm{L}^{-1}$ ). L'application de régulateurs de croissance étant indispensable pour l'enracinement des microboutures d'acacia, le témoin sans aucune hormone n'a pas été testé. Après 10 ou 20 jours de culture sur le milieu d'induction, les microboutures ont été transférées sur le milieu d'expression, de même composition que le milieu de multiplication mais sans régulateur de croissance. Pour l'ensemble des milieux testés le $\mathrm{pH}$ est ajusté à 5,8 avant apport de l'agar et autoclavage à $110^{\circ} \mathrm{C}$ pendant $20 \mathrm{~min}$.

Toutes les cultures ont été conduites dans des tubes en verre borosilicaté avec $20 \mathrm{ml}$ de milieu de culture stérile et sous une photopériode de $16 \mathrm{~h} / 8 \mathrm{~h}$, une thermopériode de $30{ }^{\circ} \mathrm{C} / 27{ }^{\circ} \mathrm{C} \pm 0,25{ }^{\circ} \mathrm{C}$, une intensité lumineuse de $50 \mu \mathrm{E} \mathrm{s}^{-1} \mathrm{~m}^{-2}$ et une hygrométrie relative $70 \pm 5 \% \mathrm{HR}$.

\section{Observations et analyse statistique des résultats}

Les variables observées (variables dépendantes) sont le nombre (NBRA) et la longueur des racines (LGRA) ainsi que la longueur de la tige (LGTI), le nombre de nœuds néoformés (NBND) et la taille du cal cicatriciel basal (CAL) de chaque vitroplant. Ces mesures ont été faites après $7,14,21$ et 28 jours de culture sur milieu d'expression.

Les traitements étudiés (variables indépendantes) sont d'une part les deux durées de l'induction de l'enracinement 
et d'autre part les régulateurs de croissance avec six niveaux différents.

Pour chaque traitement, 96 microboutures uninodales ont été utilisées. Les traitements ont été discriminés par comparaison multiple des moyennes après analyse de variance suivie du test de Newman et Keuls au seuil de $5 \%$ ou bien par test $\mathrm{Chi}^{2}$ de Pearson (logiciel Statistica).

\section{RÉSULTATS}

\subsection{Effet de la durée d'induction}

Les caractères de croissance des vitroplants qui ont subi 10 jours ou 20 jours d'induction hormonale puis 28 jours de croissance sur milieu d'expression sans hormone sont rapportés dans le tableau I. Dans tous les cas les microboutures qui ont subi une induction courte de 10 jours montrent une croissance significativement supérieure $(P<0,05)$. Le volume de l'enracinement est très nettement augmenté tant par le nombre moyen de racines par vitroplant (NBRA $=5,5$ racines pour 10 jours d'induction contre 2,4 pour 20 jours d'induction) que par leur longueur (respectivement LGRA $=2,2 \mathrm{~cm}$ en moyenne contre $1 \mathrm{~cm})$.

\subsection{Fréquence d'enracinement et croissance des pousses et des racines après 10 jours d'induction et 28 jours d'expression}

Pour l'étude détaillée de l'expression de l'enracinement, seuls les résultats obtenus à partir des vitroplants qui ont subi un traitement inductif de 10 jours sont présentés ici. Dans le tableau II sont reportés les effectifs d'explants enracinés et les fréquences d'enracinement en fonction de la nature de la balance hormonale utilisée dans le milieu d'induction. Le pourcentage moyen d'enracinement est de $56 \%$ et atteint $80 \%$ pour les vitroplants traités à l' AIB à $10 \mathrm{mg} \mathrm{L}^{-1}$. Le pourcentage le plus faible, $31 \%$ de vitroplants enracinés, est obtenu avec le traitement à l'ANA à $20 \mathrm{mg} \mathrm{L}^{-1}$.

Les vitroplants enracinés après un traitement à base d'ANA présentent, pour $73 \%$ à $90 \%$ d'entre eux, peu de racines (1 à 5 racines). Au contraire le traitement à base d'AIB à $10 \mathrm{mg} \mathrm{L}^{-1}$ avec ou sans Kinétine induit plus de 5 racines par vitroplant pour $69 \%$ à $73 \%$ des vitroplants enracinés. Parmi les vitroplants traités à l'ANA, la meilleure combinaison associe l'ANA à $10 \mathrm{mg} \mathrm{L}^{-1}$ à la Kinétine à $0,01 \mathrm{mg} \mathrm{L}^{-1}$ (57\% de plants enracinés).

Dans le tableau III sont présentés les résultats obtenus sur la croissance des pousses et des racines des microboutures. Pour les vitroplants enracinés après 28 jours de culture sur milieu d'expression, les comparaisons de moyennes montrent des différences significatives sur les caractères de développement et principalement sur le nombre de racines produites (NBRA). Toutes les combinaisons à base d'AIB induisent le développement d'un nombre de racines plus grand que toutes les combinaisons à base d'ANA (10 à 15 en moyenne, avec un maximum > 50 racines produites). Ces racines sont toutefois plagiotropes et se développent à partir d'un cal basal souvent important (CAL). Au contraire, les racines qui se développent en présence d'ANA sont plus longues (4,6 à $5,0 \mathrm{~cm}$ en moyenne) et peu nombreuses (4 racines en moyenne). Elles sont plus vigoureuses et de type orthotrope. Parallèlement l'ANA permet une croissance de la

Tableau I. Effet de la durée d'induction (10 jours ou 20 jours) sur la croissance des tiges et des racines de vitroplants d'Acacia raddiana 28 jours après transfert sur milieu d'expression sans hormone.

\begin{tabular}{|c|c|c|c|c|c|c|}
\hline \multirow{2}{*}{$\begin{array}{l}\text { Variable de } \\
\text { croissance }\end{array}$} & \multicolumn{2}{|c|}{ Durée d'induction } & \multicolumn{4}{|c|}{ Analyse de variance } \\
\hline & 10 jours & 20 jours & ddl & $\mathrm{CM}$ & $\mathrm{F}$ & $\mathrm{P}$ \\
\hline LGTI (cm) & $1,0^{\mathrm{a}(1)}$ & $0,7^{\mathrm{b}}$ & 1 & 22,9 & 21,7 & $<0,05$ \\
\hline NBND & $2,4^{\mathrm{a}}$ & $2,0^{\mathrm{b}}$ & 1 & 35,5 & 11,5 & $<0,05$ \\
\hline LGRA $(\mathrm{cm})$ & $2,2^{\mathrm{a}}$ & $1,0^{\mathrm{b}}$ & 1 & 338,8 & 56 & $<0,05$ \\
\hline NBRA & $5,5^{\mathrm{a}}$ & $2,4^{\mathrm{b}}$ & 1 & 2365,6 & 41,4 & $<0,05$ \\
\hline CAL & $0,9^{\mathrm{b}}$ & $1,3^{\mathrm{a}}$ & 1 & 47,4 & 53,7 & $<0,05$ \\
\hline Effectif & 566 & Total $=1132$ & & & & \\
\hline
\end{tabular}

LGTI = longueur moyenne des tiges en $\mathrm{cm} ; \mathrm{NBND}=$ nombre moyen de nœuds $;$ LGRA = longueur moyenne des racines en $\mathrm{cm}$; NBRA = nombre moyen des racines ; $\mathrm{CAL}=$ taille du cal basal en unité arbitraire déterminée sur une échelle de 0 à $3: 0=$ absence de cal, $1=$ petit cal, $2=$ cal moyen, $3=$ cal important.

(1) : Sur une même ligne les lettres indiquent les groupes significativement différents au seuil de $5 \%$. 
Tableau II. Influence des régulateurs de croissance appliqués pendant 10 jours d'induction sur l'abondance des racines de microboutures d'Acacia raddiana 28 jours après transfert sur milieu d'expression sans hormone.

\begin{tabular}{|c|c|c|c|c|c|c|c|c|c|}
\hline \multicolumn{10}{|c|}{ Régulateur de croissance appliqué pendant l'induction (en mg L ${ }^{-1}$ ) } \\
\hline & ANA10 & $\begin{array}{c}\text { ANA10 } \\
+ \text { kin } 0,01\end{array}$ & ANA20 & AIB10 & $\begin{array}{c}\text { AIB } 10 \\
+ \text { kin } 0,01\end{array}$ & AIB20 & Total ANA & Total AIB & Total \\
\hline Effectif & 96 & 96 & 94 & 96 & 91 & 93 & 286 & 280 & 566 \\
\hline $\mathrm{NBRA}=0$ & 62 & 41 & 65 & 19 & 29 & 34 & 168 & 82 & 250 \\
\hline Pcent & $65 \%$ & $43 \%$ & $69 \%$ & $20 \%$ & $32 \%$ & $36 \%$ & $59 \%$ & $29 \%$ & $44 \%$ \\
\hline NBRA $>0$ & 34 & 55 & 29 & 77 & 62 & 59 & 118 & 198 & 316 \\
\hline Pcent & $35 \%{ }^{c}$ & $57 \%$ & $31 \%^{c}$ & $80 \%^{\mathrm{a}}$ & $68 \%$ b & $63 \%$ & $41 \%$ & $71 \%$ & $56 \%$ \\
\hline $\mathrm{NBRA}=1$ à 5 & 28 & 40 & 26 & 21 & 19 & 24 & 94 & 64 & 158 \\
\hline Pcent $^{(1)}$ & $82 \%$ & $73 \%$ a & $90 \%$ & $27 \%$ b & $31 \%$ & $41 \%{ }^{b}$ & $80 \%$ & $32 \%$ & $50 \%$ \\
\hline NBRA > 5 & 6 & 15 & 3 & 56 & 43 & 35 & 24 & 134 & 158 \\
\hline Pcent $^{(1)}$ & $18 \%$ & $27 \%$ & $10 \%$ & $73 \%$ & $69 \%$ & $59 \%$ & $20 \%$ & $68 \%$ & $50 \%$ \\
\hline
\end{tabular}

Sur une même ligne les lettres indiquent les groupes significativement différents au seuil de $5 \%$.

${ }^{(1)}$ Les pourcentages sont calculés sur l'effectif NBRA $>0$.

Tableau III. Effet des régulateurs de croissance utilisés pendant 10 jours d'induction sur la croissance des tiges et des racines de vitroplants d'Acacia raddiana 28 jours après transfert sur milieu d'expression sans hormone.

\begin{tabular}{|c|c|c|c|c|c|c|c|c|c|c|}
\hline \multirow{2}{*}{$\begin{array}{l}\text { Variables de } \\
\text { croissance }\end{array}$} & \multicolumn{6}{|c|}{ Balance hormonale $\left(\mathrm{mg} \mathrm{L}^{-1}\right)$} & \multicolumn{4}{|c|}{ Analyse de variance } \\
\hline & ANA10 & $\begin{array}{c}\text { ANA10 } \\
+ \text { kin } 0,01\end{array}$ & ANA20 & AIB10 & $\begin{array}{c}\text { AIB10 } \\
+ \text { kin } 0,01\end{array}$ & AIB20 & ddl & $\mathrm{CM}$ & $\mathrm{F}$ & $\mathrm{P}$ \\
\hline LGTI (cm) & $1,5^{\mathrm{ab}(1)}$ & $1,7^{\mathrm{bc}}$ & $2,2^{c}$ & $1,0^{\mathrm{ab}}$ & $1,0^{\mathrm{ab}}$ & $0,9^{\mathrm{a}}$ & 5 & 11,7 & 6 & $<0,05$ \\
\hline NBND & $3,5^{\mathrm{b}}$ & $3,5^{\mathrm{b}}$ & $3,7^{\mathrm{b}}$ & $2,3^{\mathrm{a}}$ & $2,7^{\mathrm{ab}}$ & $2,3^{\mathrm{a}}$ & 5 & 21,0 & 4,8 & $<0,05$ \\
\hline LGRA (cm) & $4,9^{\mathrm{c}}$ & $4,6^{b c}$ & $5,0^{c}$ & $3,7^{\mathrm{abc}}$ & $3,4^{\mathrm{ab}}$ & $3,1^{\mathrm{a}}$ & 5 & 29,4 & 4,4 & $<0,05$ \\
\hline NBRA & $4,0^{\mathrm{a}}$ & $4,4^{\mathrm{a}}$ & $3,8^{\mathrm{a}}$ & $14,2^{\mathrm{b}}$ & $14,8^{b}$ & $10,4^{\mathrm{b}}$ & 5 & 1366,1 & 11,9 & $<0,05$ \\
\hline CAL & $0,2^{\mathrm{a}}$ & $0,6^{\mathrm{a}}$ & $0,6^{\mathrm{a}}$ & $1,5^{\mathrm{b}}$ & $1,3^{\mathrm{b}}$ & $1,4^{\mathrm{b}}$ & 5 & 14,8 & 18,4 & $<0,05$ \\
\hline $\begin{array}{l}\text { Effectifs des plants } \\
\text { enracinés }\end{array}$ & 34 & 55 & 29 & 77 & 62 & 59 & & & & \\
\hline
\end{tabular}

LGTI = longueur moyenne des tiges en $\mathrm{cm} ; \mathrm{NBND}=$ nombre moyen des nœuds $;$ LGRA = longueur moyenne des racines en $\mathrm{cm} ; \mathrm{NBRA}=$ nombre moyen des racines $; \mathrm{CAL}=$ taille du cal basal en unité arbitraire déterminée sur une échelle de 0 à $3: 0=$ absence de cal $; 1=$ petit cal $; 2=$ cal moyen $; 3=$ cal important..

${ }^{(1)}$ Sur une même ligne les lettres indiquent les groupes significativement différents au seuil de $5 \%$.

tige (LGTI) meilleure que l'AIB à $20 \mathrm{mg} \mathrm{L}^{-1}$, en particulier ANA à $20 \mathrm{mg} \mathrm{L}^{-1}$ ou ANA à $10 \mathrm{mg} \mathrm{L}^{-1}$ associé à la kinétine à $0,01 \mathrm{mg} \mathrm{L}^{-1}$ (accroissement de 1,7 à $2,2 \mathrm{~cm}$ en moyenne en 28 jours).

\subsection{Cinétique de croissance des racines}

La figure 1 montre la cinétique de croissance des racines exprimée par le nombre de racines par explant $(f i$ gure $1 a$ ) et la longueur moyenne des racines par explant (figure $1 b$ ), à partir du transfert des microboutures sur milieu d'expression après une induction de 10 jours. Dans nos conditions expérimentales, nous avons observé que quelle que soit la durée du traitement inductif appliqué, les premières racines n'apparaissent qu'après le transfert des explants sur milieu d'expression. On observe que sur les milieux à base d'ANA, le nombre de racines produites est proche de la valeur maximum (3 racines, $\mathrm{SE}=0,25$ ) dès le septième jour de culture sur le milieu d'expression. Dans le même temps, le traitement à 1 'AIB à $10 \mathrm{mg} \mathrm{L}^{-1}$ combiné ou non avec la 

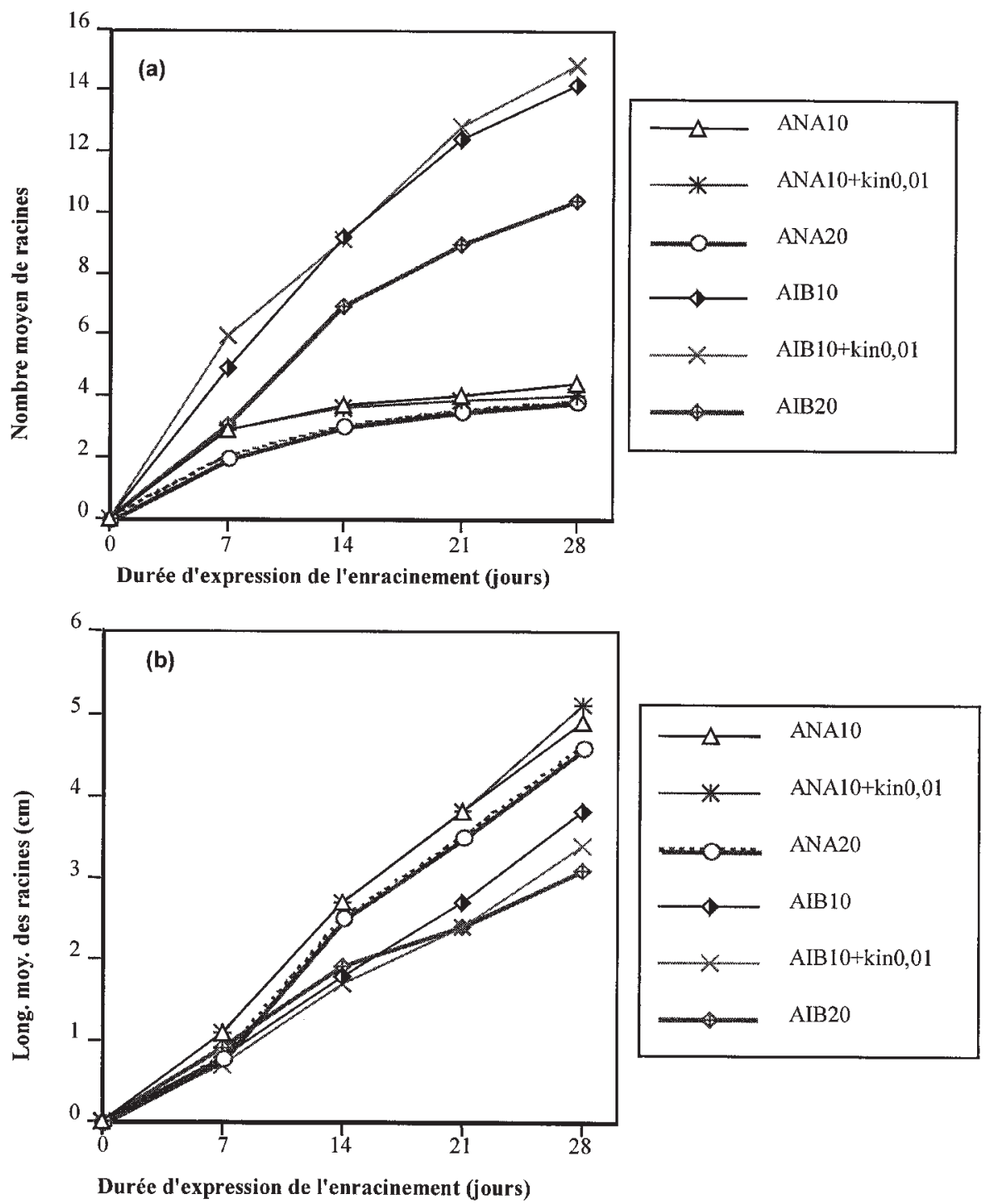

Figure 1. Cinétique de croissance des racines de microboutures d'Acacia raddiana sur milieu d'expression sans hormone après 10 jours d'induction en présence de ANA ou AIB (10 et $\left.20 \mathrm{mg} \mathrm{L}^{-1}\right)$ avec ou sans kinétine $\left(0,01 \mathrm{mg} \mathrm{L}^{-1}\right)$.

kinétine à $0,01 \mathrm{mg} \mathrm{L}^{-1}$ a déjà conduit respectivement à la formation de 5,8 et 4,8 racines en moyenne ( $\mathrm{SE}=0,25$ ). Ce nombre continue de progresser jusqu'à la fin de l'expérience (14 racines en moyenne, $\mathrm{SE}=0,60$ ).

La nature de l'auxine (AIB ou ANA) induit des différences de vitesse de croissance des racines pendant l'expérience (figure $1 b$ ). On remarque, en particulier pendant la deuxième semaine de culture, que la pente des courbes de croissance des racines est plus forte après une induction à l'ANA qu'après une induction à l'AIB. Les diffé- rences de longueur moyenne des racines sont significatives dès le $14^{\mathrm{e}}$ jour de croissance sur le milieu d'expression (d.d.1. $=5, \mathrm{CM}=10,42, \mathrm{~F}=5,81, p<0,05$ ).

\section{DISCUSSION}

Les vitroplants d'acacia utilisés dans des expériences précédentes en particulier chez A. senegal, A. raddiana et 
A. albida, perdent très rapidement leur potentialité d'enracinement $[1,3,5]$. Ainsi chez A. raddiana il a notamment été remarqué que leurs capacités à l'enracinement baissaient de près de $88 \%$ dès la deuxième subculture sur milieux de multiplication en présence de concentrations faibles ou moyennes $\left(0,1\right.$ à $\left.1,2 \mathrm{mg} \mathrm{L}^{-1}\right)$ d'ANA ou d'AIB [7]. La nécessité d'utiliser des doses d'auxine plus élevées (6 à $8 \mathrm{mg} \mathrm{L}^{-1}$ d' ANA) et surtout celle de dissocier les étapes de l'induction et de l'expression des racines a déjà été mise en évidence chez Pinus radiata [2], comme chez A. senegal [1]. Chez cette espèce, un traitement inductif de 12 jours à l'ANA à $8 \mathrm{mg} \mathrm{L}^{-1}$ apparaît meilleur qu'un traitement de 6 jours et permet une nette amélioration du taux d'enracinement des microboutures dès la deuxième subculture.

L'application transitoire d'auxine (ANA ou AIB) pendant dix jours a amélioré l'enracinement des microboutures d'A. raddiana. Le pourcentage de microboutures enracinées après leur transfert sur milieu d'expression est de $56 \%$ en moyenne et atteint $80 \%$ pour le traitement à $10 \mathrm{mg} \mathrm{L}^{-1} \mathrm{~d}^{\prime}$ AIB. Nous avons aussi observé qu' un traitement inductif de 10 jours sur milieux enrichis en AIB ou ANA s'avère meilleur qu'un traitement de 20 jours. Cependant, il fort possible qu' un traitement encore plus court (un jour ou même quelques heures) soit suffisant pour induire la rhizogenèse chez cette espèce comme cela a été montré chez le porte-greffe de pommier [13].

Les deux auxines utilisées n'ont pas la même action sur la morphologie du système racinaire mis en place. L'ANA induit la formation de racines peu nombreuses et de type orthotrope proche de la morphologie racinaire normale de cette espèce, sans formation de cal basal. L'AIB, au contraire, induit la formation d'un cal basal à partir duquel se développent de très nombreuses racines fines et plagiotropes. La même observation a été faite sur une autre légumineuse pérenne (Grevillea robusta) chez laquelle l'ANA induit des racines mieux formées que l'AIB [15]. Cependant, la formation d'un cal basal important et de racines anormales en présence d'AIB suggère que cette auxine aurait pu être en surdosage dans nos conditions expérimentales. Une nouvelle expérimentation avec des traitements hormonaux plus faibles (concentration et durée d'application) permettrait de préciser l'action réelle de l'AIB sur l'enracinement des microboutures de cette espèce.

L'application de régulateurs de croissance est nécessaire au développement des racines des microboutures mais le développement visible des racines ne commence qu'après le transfert des explants sur le milieu sans hormone et cela quelle que soit la durée du traitement induc- tif appliquée. L'ANA et l'AIB sont donc nécessaires pour induire la formation des primordiums racinaires chez A. raddiana, mais inhibent le développement des racines. La même observation a été faite chez A. senegal [1]. De même, chez Grevillea robusta, l'induction de racines a été observée sur des microboutures après traitement à l'ANA $0,27 \mu \mathrm{M}$ mais aucune racine ne s'est développée après 45 jours de culture sur le milieu avec auxine [15]. L'inhibition du développement racinaire sur milieu d'induction serait due à un métabolisme de l'auxine dont certains métabolites seraient inhibiteurs de la croissance des primordiums [14].

Dans notre expérimentation, le meilleur traitement inducteur de l'enracinement de microboutures d'A. tortilis subsp. raddiana in vitro combine l'ANA comme auxine $\left(10 \mathrm{mg} \mathrm{L}^{-1}\right)$ et une faible dose de cytokinine (kinétine à $\left.0,01 \mathrm{mg} \mathrm{L}^{-1}\right)$. Avec ce traitement les racines sont robustes et orthotropes et le taux d'enracinement atteint $57 \%$. Même si l'AIB peut induire un taux d'enracinement meilleur, le système racinaire formé avec ce régulateur de croissance pourrait être inopérant lors de la transplantation de ces arbres sahéliens en milieu naturel.

Remerciements : Ce travail a pu être réalisé grâce à une subvention de l'Institut de Recherche pour le Développement (IRD).

\section{RÉFÉRENCES}

[1] Badji S., Mairone Y., Ndiaye I., Merlin G., Danthu P., Neville P., Colonna J.P., In vitro propagation of the gum arabic tree (Acacia senegal (L) (Willd)). Developping a rapid method for producing plants, Plant Cell Reports 12 (1993) 629-633.

[2] Bergman B.A., Stomp A.-M., Effect of genotype on rooting of hypocotyls and in vitro-produced shoots of Pinus radiata, Plant Cell Tissue Organ Cult. 39 (1994) 195-202.

[3] Borgel A., Diouf M., Kparé Y., Effet de l'origine génétique sur l'aptitude au clonage in vitro d'Acacia raddiana, Bois For. Trop. 238 (1993) 23.

[4] Brenan J.P.M., Present taxonomy of four species of Acacia (A. albida, A. senegal, A. nilotica, A. tortilis), in: Manual on taxonomy of Acacia species, FAO, Rome, 1983, pp. 1-45.

[5] Duhoux E., Davies D., Caulogenèse à partir de bourgeons cotylédonaires d'Acacia albida et influence du saccharose sur la rhizogenèse, J. Plant Physiol. 121 (1985) 175-180.

[6] Duhoux E., Dommergues Y.R., The use of nitrogen fixing trees in forest and soil restoration in the tropics, in: Les arbres fixateurs d'azote et l'amélioration de la fertilité des sols. Actes Séminaire Fis/Orstom, Dakar, Sénégal, 1986, pp. 384-400. 
[7] Kparé Y.M., Influence de l'expression de la variabilité génétique sur les potentialités de clonage d'Acacia tortilis subsp. raddiana (Savi.) Brenan. Mémoire de DEA de Biologie Végétale de l'Université Cheikh Anta DIOP de Dakar, Sénégal, 1992.

[8] Lloyd G.B., McCown B.H., Commercially-feasible micropropagation of mountain-laurel, Kalmia latifolia, by use of shoot tip culture, Comb. Proc. Int. Plant Prop. Soc. 30 (1980) 412-427.

[9] Mullin T.J., Park Y.S., Estimating gains from alternative breeding strategies for clonal forestry, Can. J. For. Res. 22 (1992) 14-23.

[10] Murashige T., Skoog F., A revised medium for rapid growth and bioassays with tobacco tissue cultures, Plant Physiol. 15 (1962) 473-497.

[11] Nandwani D., In vitro propagation of a tree legume adapted to arid lands Acacia tortilis subsp. raddiana, Ann. Sci. For. 52 (1995) 183-189.
[12] Nitsch J.P., Nitsch C., Néoformation de fleurs in vitro chez une espèce de jours courts : Plumbago indica, Ann. Phys. Veg. 7 (1965) 251-256.

[13] Pawlicki N., Welander M., Influence of carbohydrate source, auxin concentration and time of exposure on adventitious rooting of the apple rootstock Jork 9, Plant Sci. 106 (1995) 167-176.

[14] Quoirin M., Boxus P., Gaspar Th., Root initiation and isoperoxidases of stem tip cuttings from mature Prunus plants, Physiol. Veg. 12 (2) (1980) 165-174.

[15] Rajasekaran P., Production of clonal plantlets of Grevillea robusta in in vitro culture via axillary bud activation, Plant Cell Tissue Organ Cult. 39 (1994) 277-279.

[16] Thorpe T.A., Harry I.S., Kumar P.P., Application of micropropagation to forestry, in: Debergh P.P., Zimmerman R.H. (Eds.), Micropropagation technology and application, New York, 1991, pp. 311-336.

To access this journal online: www.edpsciences.org 
